\title{
УМНОЖЕНИЕ ЧАСТОТ ТЕРАГЕРЦОВОГО ИЗЛУЧЕНИЯ В КРИСТАЛЛАХ ПАРАЭЛЕКТРИКА ТИТАНАТА СТРОНЦИЯ
}

\author{
ЗАМУДИО-ЛАРА А., КОШЕВАЯ С. В., ГРИМАЛЬСКИЙ В. В., ЯНЬЕЗ-КОРТЕС Ф. \\ Автономный университет штата Морелос, \\ Мексика, Куэрнавака, Z. Р. 62209
}

\begin{abstract}
Аннотация. Исследовано умножение частот терагерцового излучения в кристаллах параэлектрика титаната стронция при температуре 77 К. На процесс генерации гармоник влияет частотная дисперсия. Показано, что эффективность генерации высших гармоник высокая и составляет 30\%. Имеется возможность селективного выделения определенной гармоники за счет оптимального выбора длины кристалла
\end{abstract}

Ключевые слова: ТГц; терагерцовое излучение; умножение частоты; параэлектрик

\section{1. ВВЕДЕНИЕ}

В последнее время идет интенсивное освоение терагерцового (ТГц) диапазона 0,1-30 ТГц, который нашел применение в интроскопии, спектроскопии, широкополосной связи, радиоастрономии и радиовидении [1-3]. Для генерации ТГц излучения используются источники типа оптических (лазеры, оптоэлектронные антенны) и типа СВЧ (лампы обратной волны, гиротроны, диодные генераторы) $[1,2]$. Однако эффективность источников невысока и обычно составляет не более нескольких процентов.

В ТГц диапазоне перспективно умножение частот, когда в качестве нелинейных умножителей частоты используются диоды Шоттки и диоды на гетероструктурах. В последние годы в этом направлении достигнут значительный прогресс [3-5], однако диодные умножители имеют малую электрическую прочность и требуют применения резонансных волноводных структур.

Для умножения частот СВЧ излучения, начиная с 1960 годов применяются нелинейные диэлектрики, в частности, сегнетоэлектрики в неполярной фазе, т.н. параэлектрики титанат стронция $\mathrm{SrTiO}_{3}$, танталат калия $\mathrm{KTaO}_{3}$ и керамики на их основе [6-15]. Параэлектрики характеризуются высокой электродинамической нелинейностью и малыми потерями при низких температурах [6-10]. Показана высокая эффективность нелинейного преобразования частот вверх в СВЧ диапазоне [11-15].

В последние годы изучается применение параэлектриков и композитных материалов на основе сегнетоэлектриков в ТГц диапазоне [9, 16-18]. Кристаллический $\mathrm{SrTiO}_{3}$ в низкочастотной части ТГц диапазона ниже частоты мягкой моды ( 1 ТГц) имеет высокую нелинейность и малые потери при умеренно низких температурах 50-90 К [6-8], однако в отличие от СВЧ диапазона сказывается частотная дисперсия диэлектрической проницаемости [9, 17]. Низшая оптическая (электрически активная) мода колебаний кристаллической решетки параэлектрика получила название мягкой моды, поскольку ее частота значительно ниже других оптических колебаний кристалла [6-8]. 
Для кристалла $\mathrm{SrTiO}_{3}$ частота мягкой моды убывает с температурой [6]. При температуре перехода параэлектрик-сегнетоэлектрик, например, для титаната бария $\mathrm{BaTiO}_{3}$, частота мягкой моды стремится к нулю.

В данной работе рассмотрена генерация высших гармоник миллиметрового и ТГц излучения в кристаллическом параэлектрике титанате стронция при наличии постоянного электрического поля смещения, когда доминирующей нелинейностью является квадратичная. Рассмотрен случай азотного уровня охлаждения 77 К. Показана высокая эффективность генерации высших гармоник, исследовано влияние частотной дисперсии и указана возможность селективно возбуждать определенный номер гармоники в зависимости от длины кристалла. Имеется оптимум эффективности преобразования по электрическому полю смещения.

\section{2. СВОЙСТВА ПАРАЭЛЕКТРИКОВ В ТГЦ ДИАПАЗОНЕ И ОСНОВНЫЕ ФОРМУЛЫ}

Используем следующую формулу для линейной диэлектрической проницаемости параэлектриков, которая справедлива в СВЧ и ТГц диапазонах $[6,9]$ :

$$
\begin{gathered}
\varepsilon(\omega)=\frac{B}{\omega_{T}^{2}-\omega^{2}+i \gamma \omega}, \quad \omega>0, \\
B=\varepsilon(\omega=0) \omega_{T}^{2},
\end{gathered}
$$

где $\omega_{T}$ - частота мягкой моды, которая для $\mathrm{SrTiO}_{3}$ находится в ТГц диапазоне, $\gamma$ - решеточное затухание, $\mathrm{i}$ - мнимая единица.

Частота мягкой моды растет с ростом температуры $T: \omega_{T} \sim T$. При температуре $77 \mathrm{~K}$ ее величина равна примерно $\omega_{T} \approx 6 \times 10^{12} \mathrm{c}^{-1}$, а статическая диэлектрическая проницаемость равна $\varepsilon(\omega=0)=1,8 \times 10^{3}$. Для титаната стронция диэлектрическая проницаемость растет с уменьшением температуры.
Зависимость диэлектрической проницаемости от электрического поля имеет вид [6]:

$$
\varepsilon(E, \omega=0)=\frac{B}{\omega_{T}^{2}\left(1+\frac{3 E^{2}}{E_{0}^{2}}\right)^{1 / 3}},
$$

где $E_{0}$ - электрическое поле, которое характеризует нелинейность: $E_{0} \sim(\varepsilon(\omega=0))^{-3 / 2}[6,10$, 11]. Таким образом, нелинейность, пропорциональная $E_{0}^{-2}$, растет пропорционально кубу диэлектрической проницаемости.

На основании (1), (2) представим комбинированную приближенную формулу для диэлектрической проницаемости, которая зависит от частоты и электрического поля:

$$
\begin{gathered}
\varepsilon(E, \omega) \approx \frac{B}{\omega_{T}^{2}\left(1+\frac{E^{2}}{E_{0}^{2}}\right)-\omega^{2}+\mathrm{i} \gamma \omega}, \\
\omega<\omega_{T}, \quad|E|<E_{0} .
\end{gathered}
$$

Формула (3) справедлива при частотах ниже частоты мягкой моды $\omega<\omega_{T}$. В случае $\omega \geq \omega_{T}$ следует использовать отдельное уравнение для электрической поляризации.

Рассмотрим генерацию гармоник линейно поляризованной электромагнитной волны высокочастотной части миллиметрового и низкочастотной части ТГц диапазонов:

$$
E \equiv E_{x}=E_{s}+\widetilde{E}, \quad|\widetilde{E}|<<E_{s}<E_{0},
$$

где $E_{s}$ - постоянное поле смещения, $\widetilde{E}$ - поле электромагнитной волны. Волна распространяется вдоль оси $O z$ и поляризована вдоль оси $O x$.

Из (3) получаем выражение для переменной электрической индукции электромагнитной волны (ее положительных частот): 


$$
\begin{gathered}
\widetilde{D}=\varepsilon_{0} \frac{B}{\Delta}\left[\left(1-\frac{2 \omega_{T}^{2}}{\Delta} \frac{E_{s}}{E_{0}}-\frac{\mathrm{i} \gamma \omega}{\Delta}\right) \widetilde{E}-\frac{3 \omega_{T}^{2} E_{s}}{\Delta} \frac{\widetilde{E}^{2}}{E_{0}^{2}}\right], \\
\Delta \equiv \omega_{T}^{2}\left(1+\frac{E_{s}^{2}}{E_{0}^{2}}\right)-\omega^{2}+\mathrm{i} \gamma \omega
\end{gathered}
$$

где $\varepsilon_{0}$ - электрическая постоянная.

Таким образом, линейная часть эффективной проницаемости с учетом затухания при наличии поля смещения $E_{s}$ имеет вид:

$$
\varepsilon_{s}(\omega)=\frac{B}{\Delta}\left(1-\frac{2 \omega_{T}^{2}}{\Delta} \frac{E_{s}}{E_{0}}-\frac{\mathrm{i} \gamma \omega}{\Delta}\right) .
$$

Волновое уравнение для электромагнитной волны представим в виде:

$$
\frac{\partial^{2} \widetilde{E}}{\partial z^{2}}-\frac{1}{c^{2} \varepsilon_{0}} \frac{\partial^{2} \widetilde{D}}{\partial t^{2}}=0 .
$$

Решение волнового уравнения определяется как набор гармоник [11-15]:

$$
\begin{gathered}
\widetilde{E}=\frac{1}{2} \sum_{j=1,2, \ldots}\left(A_{j}(z) \exp \left(\mathrm{i} \varphi_{j}\right)+A_{j}^{*}(z) \exp \left(-\mathrm{i} \varphi_{j}\right)\right), \\
\varphi_{j}=j\left(\omega_{1} t-k_{1} z\right), \omega_{j} \equiv j \omega_{1}, \\
k_{j}=\frac{\omega_{j}}{c} \operatorname{Re}\left(\left(\varepsilon_{s}\left(\omega_{j}\right)\right)^{1 / 2}\right),
\end{gathered}
$$

где $A_{j}(z)$ - медленно изменяющиеся амплитуды, $\left|\partial A_{j} / \partial z\right|<<k_{j}\left|A_{j}\right|, k_{j}$ - волновые числа гармоник, $j=1,2,3 \ldots$

Далее рассмотрен стационарный случай $A_{j}(z)$. Однако умножение частоты следует проводить в импульсном режиме из-за возможного нагрева кристалла.

Используем метод медленно изменяющихся амплитуд, который заключается в следующем $[11,19]$. При подстановке (8) в волновое уравнение (7) вторая производная приближенно вычисляется как [11]:

$$
\begin{gathered}
\frac{\partial^{2} \widetilde{E}}{\partial z^{2}} \approx-\frac{1}{2} \sum_{j=1,2, \ldots}\left(j k_{1}\right)^{2}\left(A_{j}(z) \exp \left(\mathrm{i} \varphi_{j}\right)+\right. \\
\left.+A_{j}^{*}(z) \exp \left(-\mathrm{i} \varphi_{j}\right)\right)-\sum_{j=1,2, \ldots}\left(j k_{1}\right) \times \\
\times\left(\frac{\mathrm{d} A_{j}(z)}{\mathrm{d} z} \exp \left(\mathrm{i} \varphi_{j}\right)-\frac{\mathrm{d} A_{j}^{*}(z)}{\mathrm{d} z} \exp \left(-\mathrm{i} \varphi_{j}\right)\right)
\end{gathered}
$$

Приравнивая в (9) коэффициенты при несущих частотах $\omega_{1}, \omega_{2}$ и т.д., получаем систему уравнений (10) для медленно изменяющихся амплитуд (см. внизу с. 3).

$\mathrm{B}(10) \Gamma_{j} \sim \omega_{j}^{2}$ - затухание гармоник, $\Delta k_{j}$ - расстройки от точного синхронизма.

Граничные условия для данного случая генерации гармоник имеют вид:

$$
A_{1}(z=0)=A_{10}, \quad A_{j}(z=0)=0, \quad j>1 .
$$

Существует отличие генерации высших гармоник в ТГц диапазоне от данного процесса в сантиметровом диапазоне. В сантиметровом диапазоне в кристалле или волноводе создается ударная волна и возбуждается более 10 гармоник из-за отсутствия частотной дисперсии, а число гармоник ограничено затуханием [11-15]. В ТГц диапазоне сказывается частот-

$$
\left\{\begin{array}{c}
\frac{\mathrm{d} A_{1}}{\mathrm{~d} z}+\Gamma_{1} A_{1}-\frac{3 \mathrm{i} \omega_{1}\left(\varepsilon_{s}\left(\omega_{1}\right)\right)^{1 / 2}}{2 c} \frac{E_{s}}{E_{0}^{2}} \sum_{l=2,3, \ldots} A_{l} A_{l-1}^{*}=0, \\
\frac{\mathrm{d} A_{j}}{\mathrm{~d} z}+\left[\Gamma_{j}+\mathrm{i} \Delta k_{j}\right] A_{j}-\frac{3 \mathrm{i} \omega_{j}\left(\varepsilon_{s}\left(\omega_{j}\right)\right)^{1 / 2}}{4 c} \frac{E_{s}}{E_{0}^{2}}\left\{\sum_{l<j} A_{l} A_{j-l}+2 \sum_{l>j} A_{l} A_{l-j}^{*}\right\}=0, j>1, \\
\Gamma_{j}=\frac{\omega_{j}}{c} \operatorname{Im}\left(\left(\varepsilon_{s}\left(\omega_{l}\right)\right)^{1 / 2}\right), \quad \Delta k_{j}=k_{j}-j k_{1},
\end{array}\right.
$$




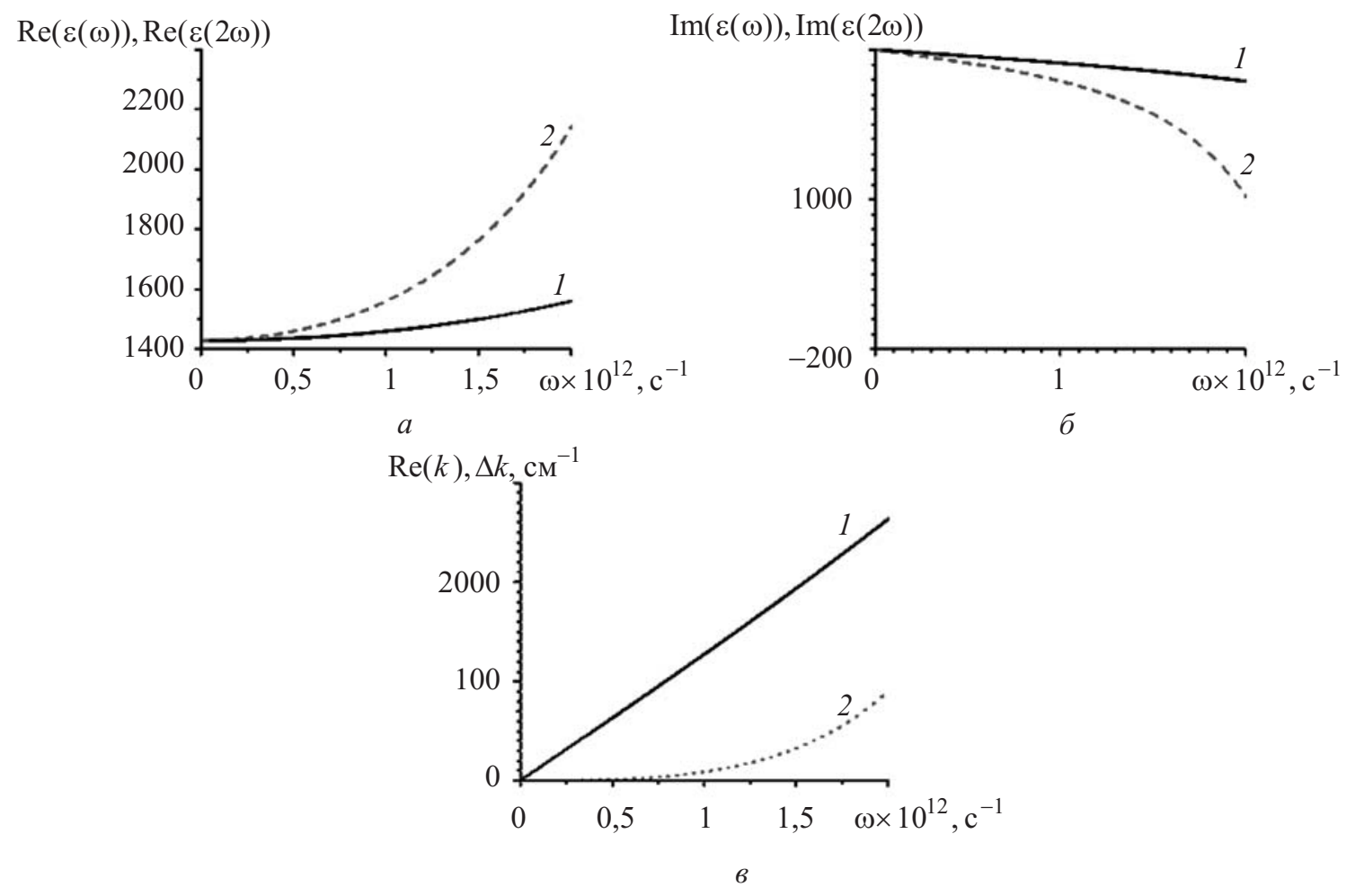

Рис. 1

ная дисперсия, поэтому число гармоник реально не более 5-6. Более высокие гармоники не возбуждаются из-за большой расстройки.

\section{3. ЭФФЕКТИВНОСТЬ ПРЕОБРАЗОВАНИЯ}

В расчетах эффективности умножения частот использованы следующие параметры $\mathrm{SrTiO}_{3}$ при температуре $T=77 \mathrm{~K}$ : характеристическое поле $E_{0}=6 \times 10^{4} \mathrm{~B} / \mathrm{cm}$, частота мягкой моды $\omega_{T}=6 \times 10^{12} \mathrm{c}^{-1}$, решеточное затухание $\gamma=2 \times 10^{11} \mathrm{c}^{-1}[6,9]$. При более низких температурах характеристическое поле $E_{0}$ понижается, т.е. увеличивается нелинейность, однако при этом понижается и частота мягкой моды. Поэтому для преобразования частот в ТГц диапазоне нецелесообразно использовать более низкие температуры.

Поскольку с ростом поля смещения $E_{s}$ эффективная диэлектрическая проницаемость уменьшается (см. (3)), то существует оптимальная величина поля смещения. Проведен- ные расчеты показали, что его величина составляет $E_{s} \approx 0,3 E_{0}$. При расчетах использовалось значение $E_{s}=0,3 E_{0}=1,8 \times 10^{4} \mathrm{~B} / \mathrm{cm}$.

Численные расчеты умножения частот в кристалле титаната стронция (рис. 1-4) показали высокую эффективность этого процесса. При этом существенна роль частотной дисперсии, и, как следствие, расстройки от точного синхронизма. На рис. 1 представлены зависимости от частоты действительных (рис. 1a) и мнимых (рис. 1б) частей эффективной диэлектрической проницаемости для первой (кривая 1) и второй (кривая 2) гармоник, а также зависимости действительной части волнового числа (рис. 1в) для первой гармоники (кривая 1) и расстройки (кривая 2).

Распределения квадратов амплитуд для гармоник по длине кристалла представлены на рис. 2, 3, 4 (общий вид на рис. $2 a-4 a$, вид в масштабе на рис. 2б-4б). Для всех рисунков значение амплитуды первой гармоники на входе 

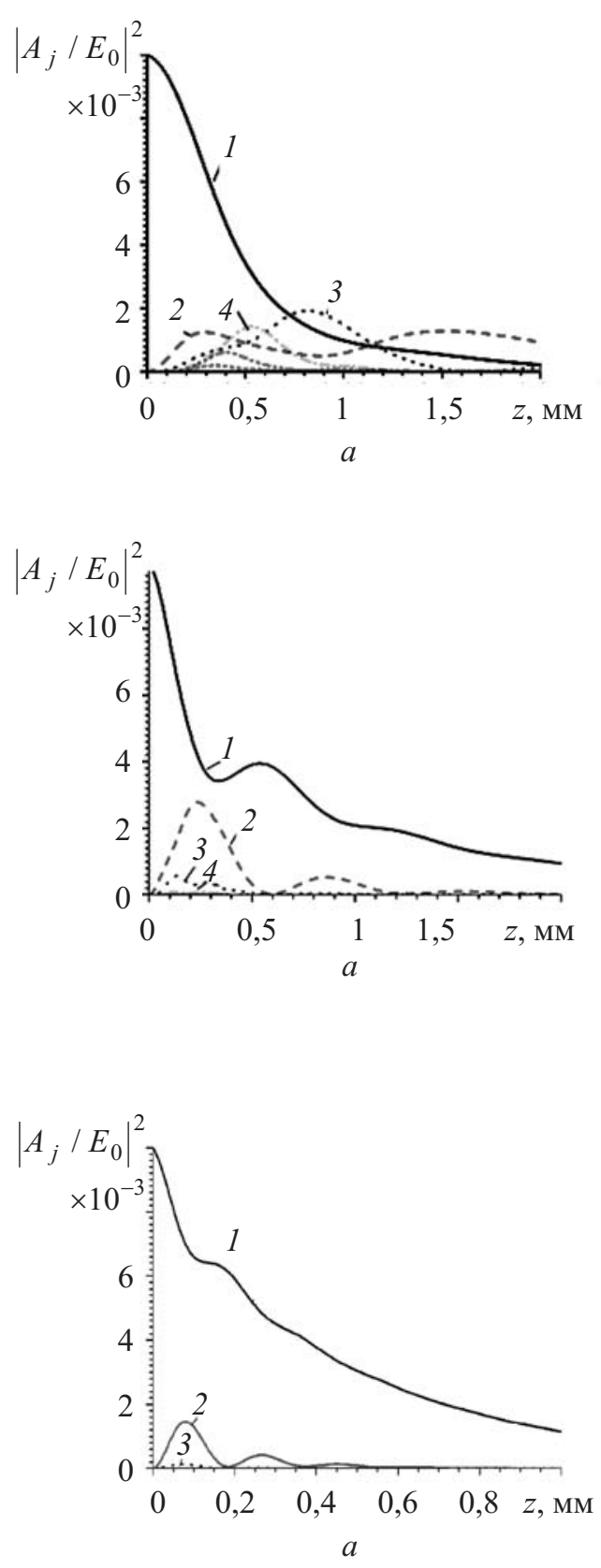

равно $A_{10}=0,1 E_{0}=6 \times 10^{3} \mathrm{~B} / \mathrm{cm}$. При этом изменялась частота первой гармоники - $\omega_{1}=$ $0,5 \times 10^{12} \mathrm{c}^{-1}$ (рис. 2), $\omega_{1}=1 \times 10^{12} \mathrm{c}^{-1}$ (рис. 3), $\omega_{1}=$ $1,5 \times 10^{12} \mathrm{c}^{-1}$ (рис. 4). Видно, что при относительно малых частотах (рис. 2) в кристалле возбуждаются 5 гармоник. В этом случае имеется возможность за счет выбора длины кри-

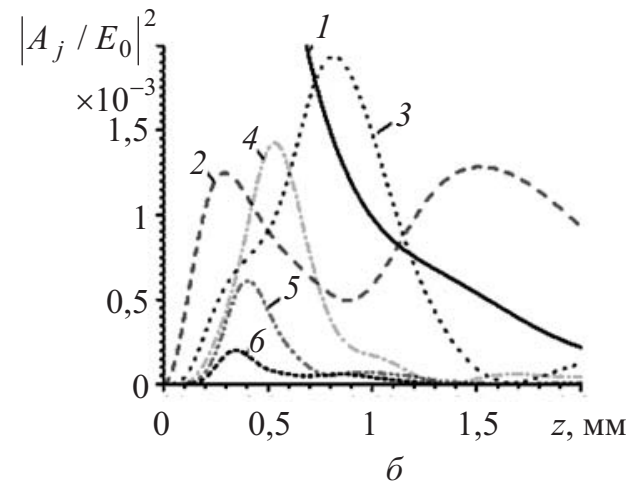

Рис. 2

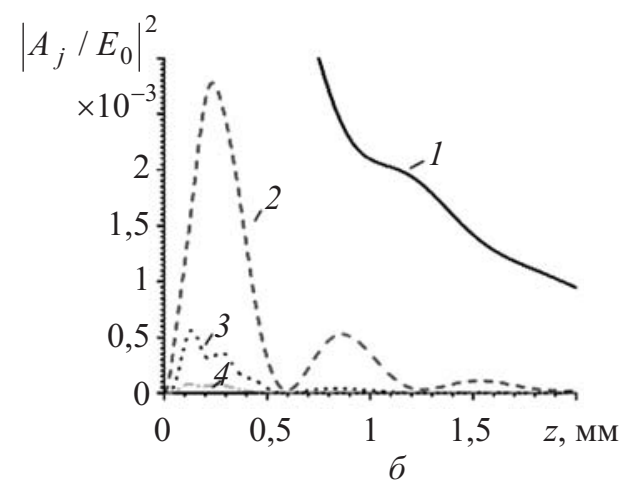

Рис. 3

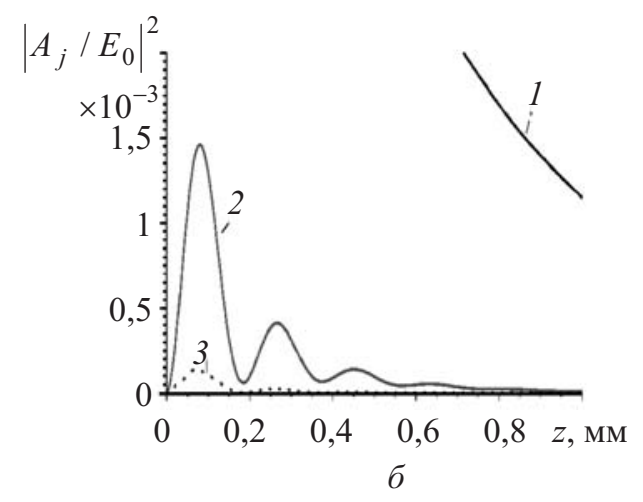

Рис. 4

сталла выделять максимумы третьей или четвертой гармоник. Например, в кристалле длиной $L_{z}=0,08$ см на выходе будет максимум третьей гармоники.

При более высоких частотах сказывается влияние частотной дисперсии проницаемости и возбуждаются 2-3 гармоники (рис. 3,4$)$. Эффективность преобразования во вторую гармо- 
нику составляет $30 \%$ и $15 \%$, соответственно. В отличие от диодных умножителей, нелинейное преобразование частоты происходит в объеме кристалла, следовательно не возникает проблем с электрической прочностью.

Проблема ввода энергии в кристалл с высокой диэлектрической проницаемостью и вывода энергии высших гармоник может быть решена с помощью четвертьволновых согласующих трансформаторов, аналогично просветлению в оптике [20]. Для широкополосного согласования возможно использовать многослойные структуры на входе и выходе из нелинейного кристалла. Эффективность согласования в СВЧ диапазоне для параэлектриков продемонстрирована в [21].

При наличии постоянного поля смещения $E_{s}$ роль кубичной нелинейности незначительна. Однако в отсутствие $E_{s}$ проявляется только кубичная нелинейность. При этом, благодаря частотной дисперсии в кристалле титаната стронция возможно распространение солитонов огибающей и модуляционной неустойчивости протяженного импульса ТГц диапазона. Для наблюдения этих эффектов следует выбирать частоту электромагнитной волны так, чтобы для третьей гармоники расстройка от синхронизма была достаточно большая. Модуляционная неустойчивость приводит к генерации последовательности коротких импульсов ТГц диапазона с длительностями порядка $0,1 \mathrm{Hc}$.

\section{4. ВЫвОДЫ}

Аналогично СВЧ диапазону, кристаллы параэлектрика титаната стронция при температуре 77 К могут использоваться для умножения частоты в ТГц диапазоне. В отличие от СВЧ диапазона, при генерации высших гармоник электромагнитной волны сказывается частотная дисперсия. Эффективность преобразования во вторую гармонику может достигать $30 \%$. Имеется возможность за счет выбора длины кристалла выделять максимумы третьей или четвертой гармоник.

\section{БИБЛИОГРАФИЧЕСКИЙ СПИСОК}

1. Lee Y.-S. Principles of Terahertz Science and Technology / Yun-Shik Lee. — N.Y. : Springer, 2009. 340 p. — DOI : 10.1007/978-0-387-09540-0.

2. Perenzoni M. (ed.). Physics and Applications of Terahertz Radiation / Ed. by Matteo Perenzoni, Douglas J. Paul. - N.Y. : Springer, 2014. - 255 p. - DOI : 10.1007/ 978-94-007-3837-9.

3. Siegel P. H. Terahertz Pioneer: Erik L. Kollberg "Instrument Maker to the Stars" / P. H. Siegel // IEEE Trans. Terahertz Sci. Technol. - Sept. 2014. — Vol. 4, No. 5. - P. 538-544. - DOI : 10.1109/TTHZ.2014. 2344191.

4. Tunable broadband frequency-multiplied terahertz sources / J. S. Ward, G. Chattopadhyay, J. Gill, H. Javadi, Choonsup Lee, R. Lin, A. Maestrini, F. Maiwald, I. Mehdi, E. Schlecht, P. Siegel // Infrared, Millimeter and Terahertz Waves : 33rd Int. Conf. IRMMW-THz, 15-19 Sept. 2008, Pasadena, CA : proc. — IEEE, 2008. - P. 1-3. - DOI : 10.1109/ICIMW.2008.4665437.

5. Лебедев А.И. Физика полупроводниковых приборов / А. И. Лебедев. - М. : Физматлит, 2008. $488 \mathrm{c}$.

6. Сегнетоэлектрики в технике СВЧ / Под ред. О. Г. Вендик. - М. : Сов. Радио, 1979. - 272 с.

7. Рез И. С. Диэлектрики. Основные свойства и применение в электронике / И. С. Рез, Ю. М. Поплавко. - М. : Радио и Связь, 1989. — 288 с.

8. Фізичне матеріалознавство. Ч. 2: Діелектрики / Ю. М. Поплавко, Л. П. Переверзєва, С. О. Воронов, Ю. І. Якименко. - К. : НТУУ КПІ, 2007. - 391 с.

9. Gevorgian $S$. Ferroelectrics in Microwave Devices, Circuits and Systems / Spartak Gevorgian. N.Y. : Springer, 2009. - 394 p. - DOI : 10.1007/9781-84882-507-9.

10. Низкотемпературные сегнетоэлектрики: диэлектрическая проницаемость, потери и параметрические взаимодействия на сверхвысоких частотах / И. В. Иванов, И. М. Бузин, Г. В. Белокопытов, В. М. Сычев, В. Ф. Чупраков // Физика. - 1981. — Т. 24, № 8. - С. 6-27. - (Известия вузов).

11. Параметрическое и нелинейное взаимодействие электромагнитных волн в параэлектриках / Л. Г. Гассанов, С. В. Кошевая, Т. Н. Нарытник, М. Ю. Омельяненко // Радиоэлектроника. - 1978. - Т. 21, № 10. - С. 56-63. - (Известия МВиССО СССР).

12. Кочевая C. B. Генерация третьей гармоники в бездисперсионом нелинейном диэлектрике / С. В. Кошевая, Л. Г. Гассанов, М. Ю. Омельяненко // Укр. Физ. Журн. - 1980. - Т. 25, № 7. - С. 1118-1123.

13. Кочевая С. В. Влияние дисперсии в волноведущих системах с кубически нелинейными диэлектриками / С. В. Кошевая, М. В. Кононов, М. Ю. Омель- 
яненко // Радиоэлектроника. - 1985. - Т. 28, № 3. С. 53-56. - (Известия вузов).

14. Кононов М. В. Нелинейные сверхвысокочастотные волны в кристаллах / М. В. Кононов, С. В. Кошевая, М. Ю. Омельяненко // Квантовая Электроника. - 1984. - Т. 11, № 26. - С. 55-68. - (Сб. Инст. Полупроводников АН УССР).

15. Гассанов Л. Г. Об умножении частот в параэлектриках / Л. Г. Гассанов, С. В. Кошевая, М. Ю. Омельяненко // Радиотехника и электроника. - 1980. — T. 25, № 6. - C. 1238-1243.

16. Vugmeyster I. D. Development of a terahertz time-domain spectrometer optimized at $5-8 \mathrm{THz}$ and the study of surface polaritons in $\mathrm{NiO}-\mathrm{SrTiO}_{3}$ nano-composite ceramics / I. D. Vugmeyster// PhD Dissertation. - The University of Michigan, 2013. - $121 \mathrm{p}$.

17. The low-temperature infrared optical functions of $\mathrm{SrTiO}_{3}$ determined by reflectance spectroscopy and spectroscopic ellipsometry / K. Kamaras, K.-L. Barth, F. Keilmann, R. Henn, M. Reedyk, C. Thomsen, M. Cardo- na, J. Kircher, P. L. Richards, J.-L. Stehle // J. Appl. Phys. - 1995. - Vol. 78, No. 3. - P. 1235-1240. — DOI : $10.1063 / 1.360364$.

18. Tunable ferroelectric ceramic-polymer composites for sub- $\mathrm{THz}$ applications / Y. Yashchyshyn, K. Godziszewski, P. Bajurko, J. Modelski, M. Szafran, E. Bobryk, E. Pawlikowska, G. Tarapata, J. Weremczuk, R. Jachowicz // 43rd European Microwave Conf. : EuMC, 6 -10 Oct. 2013, Nuremberg, Germany. — IEEE, 2013. P. 676-679. - INSPEC : 13999560.

19. Бломберген Н. Нелинейная оптика / Н. Бломберген. - М. : Мир, 1966. - 424 с.

20. Борн М. Основы оптики / М. Борн, Э. Вольф. - М. : Наука, 1973. — 720 с.

21. Кочевая С. В. Широкополосное согласование волноводов, содержащие материалы с большими $\varepsilon$ / С. В. Кошевая, М. Ю. Омельяненко // Радиоэлектроника. - 1984. - Т. 27, № 12. - С. 70-72. - (Известия МВиССО СССР).

\section{INFORMATION ON THE ARTICLE}

\section{FREQUENCY MULTIPLICATION OF TERAHERTZ RADIATION IN THE CRYSTALS OF STRONTIUM TITANATE PARAELECTRIC}

Alvaro Zamudio-Lara, Universidad Autonoma del Estado de Morelos, Cuernavaca, Morelos, Mexico

Svetlana V. Koshevaya, svetlana@uaem.mx, Universidad Autonoma del Estado de Morelos, Cuernavaca, Morelos, Mexico

Vladimir V. Grimalsky, v_grim@yahoo.com, Universidad Autonoma del Estado de Morelos, Cuernavaca, Morelos,

Mexico

Fabiola Yañez-Cortes, Universidad Autonoma del Estado de Morelos, Cuernavaca, Morelos, Mexico

In this article we have investigated frequency multiplication in the crystals of strontium titanate paraelectric at a temperature of $77 \mathrm{~K}$. Frequency dispersion affects the process of harmonics generation. It has been shown that the efficiency of higher harmonics generation is high and it is equal to $30 \%$. One can perform the selective extraction of certain harmonic by means of an optimal choice of the crystal length

Keywords: THz; THz radiation; frequency multiplication; paraelectric

\section{REFERENCES}

1. LEE, YUN-SHIK. Principles of Terahertz Science and Technology. N.Y.: Springer, 2009, 340 p., DOI: 10.1007/9780-387-09540-0.

2. PERENZONI, MATTEO; PAUL, DOUGLAS J. (eds.), Physics and Applications of Terahertz Radiation. N.Y.: Springer, 2014, 255 p., DOI: 10.1007/978-94-007-3837-9.

3. SIEGEL, P.H. Terahertz Pioneer: Erik L. Kollberg 'Instrument Maker to the Stars'. IEEE Trans. Terahertz Sci.
Technol., Sept. 2014, v.4, n.5, p.538-544, DOI: $\underline{10.1109 /}$ TTHZ.2014.2344191.

4. WARD, J.S.; CHATTOPADHYAY, G.; GILL, J.; JAVADI, H.; LEE, CHOONSUP; LIN, R.; MAESTRINI, A.; MAIWALD, F.; MEHDI, I.; SCHLECHT, E.; SIEGEL, P. Tunable broadband frequency-multiplied terahertz sources. Proc. of 33rd Int. Conf. on Infrared, Millimeter and Terahertz Waves, IRMMW-THz, 15-19 Sept. 2008, Pasadena, CA. IEEE, 2008, p.1-3, DOI: $10.1109 /$ ICIMW.2008.4665437. 
5. LEBEDEV, A.I. Physics of Semiconductor Devices. Moscow: Fizmatlit, 2008 [in Russian].

6. Ferroelectrics in Microwave Engineering. Moscow: Sov. Radio, 1979 [in Russian, ed. by O. G. Vendik].

7. REZ, I.S.; POPLAVKO, Y.M. Dielectrics. Fundamental Properties and Application in Electronics. Moscow: Radio i Svyaz', 1989 [in Russian].

8. POPLAVKO, Y.M.; PEREVERZEVA, L.P.; VORONOV, S.O.; YAKYMENKO, Y.I. Material Physics. Part 2. Dielectrics. Kyiv: NTUU KPI, 2007 [in Ukrainian].

9. GEVORGIAN, SPARTAK. Ferroelectrics in Microwave Devices, Circuits and Systems. N.Y.: Springer, 2009, 394 p., DOI: $10.1007 / 978-1-84882-507-9$.

10. IVANOV, I.V.; BUZIN, I.M.; BELOKOPYTOV, G.V.; SYCHEV, V.M.; CHUPRAKOV, V.F. Low temperature ferroelectrics: Dielectric nonlinearity, losses, and parametric interactions at ultrahigh frequencies. Soviet Physics Journal, 1981, v.24, n.8, p.684-704, DOI: 10.1007/BF00941340.

11. GASSANOV, L.G.; KOSHEVAYA, S.V.; NARYTNIK, T.N.; OMELIANENKO, M.Y. Parametric and nonlinear interaction of electromagnetic waves in paraelectrics. Radioelectron. Commun. Syst., 1978, v.21, n.10, p.52-59.

12. KOSHEVAYA, S.V.; GASSANOV, L.G.; OMELIANENKO, M.Y. Generation of the third harmonic in dispersion-free nonlinear dielectric. Ukr. J. Phys., 1980, v.25, n.7, p.1118-1123.

13. KOSHEVAYA, S.V.; KONONOV, M.V.; OMELIANENKO, M.Y. The influence of dispersion in waveguide systems with cubic nonlinear dielectrics. Radioelectron. Commun. Syst., 1985, v.28, n.3, p.48-50.

14. KONONOV, M.V.; KOSHEVAYA, S.V.; OMELIANENKO, M.Y. Nonlinear microwaves in crystals. $S b$.
Kvantovaya Elektronika. Inst. Poluprovodnikov AN USSR, 1984, n.26, p.55-68.

15. GASSANOV, L.G.; KOSHEVAYA, S.V.; OMELIANENKO, M.Y. Frequency multiplication in paraelectrics. Radiotekh. Electron., 1980, v.25, n.6, p.1238-1243. 16. VUGMEYSTER, I.D. Development of a terahertz time-domain spectrometer optimized at $5-8 \mathrm{THz}$ and the study of surface polaritons in NiO-SrTiO3 nano-composite ceramics. PhD Dissertation. The University of Michigan, 2013, $121 \mathrm{p}$.

17. KAMARAS, K.; BARTH, K.-L.; KEILMANN, F.; HENN, R.; REEDYK, M.; THOMSEN, C.; CARDONA, M.; KIRCHER, J.; RICHARDS, P.L.; STEHLE, J.-L. The low-temperature infrared optical functions of $\mathrm{SrTiO}_{3}$ determined by reflectance spectroscopy and spectroscopic ellipsometry. $J$. Appl. Phys., 1995, v.78, n.3, p.1235-1240, DOI: $\underline{10.1063 /}$ 1.360364 .

18. YASHCHYSHYN, Y.; GODZISZEWSKI, K.; BAJURKO, P.; MODELSKI, J.; SZAFRAN, M.; BOBRYK, E.; PAWLIKOWSKA, E.; TARAPATA, G.; WEREMCZUK, J.; JACHOWICZ, R. Tunable ferroelectric ceramic-polymer composites for sub-THz applications. Proc. of 43rd European Microwave Conf., EuMC, 6-10 Oct. 2013, Nuremberg, Germany. IEEE, 2013, p.676-679, INSPEC: 13999560.

19. BLOEMBERGEN, N. Nonlinear Optics. World Scientific, 1996.

20. BORN, M.; WOLF, E. Principles of Optics. Cambridge University Press, 1999.

21. KOSHEVAYA, S.V.; OMELIANENKO, M.Y. Wideband matching of waveguides containing materials with high ع. Radioelectron. Commun. Syst., 1984, v.27, n.12, p.65-68.

Received in final form April 27, 2015 